\title{
Evidence of genetic isolation between sexually monomorphic and sexually dimorphic traits in the water strider Aquarius remigis
}

\author{
RICHARD F. PREZIOSI* + \& DEREK A. ROFF \\ $\dagger$ Department of Entomology, S-225 Ag. Sci. Center North, University of Kentucky, Lexington, KY 40546-0091, U.S.A. \\ and \$Department of Biology, McGill University, Montreal, Quebec, Canada H3A 1B1
}

\begin{abstract}
Estimates of multivariate selection are becoming increasingly common in the literature. Although it is clearly recognized that genetic information is essential to understanding natural selection, few studies include estimates of genetic parameters. Previous studies of the water strider Aquarius remigis have demonstrated selection on body size and components of body size that may favour the evolution and maintenance of sexual size dimorphism. In this paper we present quantitative genetic estimates of heritabilities and genetic correlations for male and female $A$. remigis. Overall body size (total length) and the majority of components of body size were significantly heritable for both sexes and the shape of the genetic correlation matrix was similar between the sexes. However, patterns of genetic correlations between the sexes and analysis of genetic integration suggest that some traits may be genetically isolated.
\end{abstract}

Keywords: genetic correlation, genetic integration, heritability, size dimorphism.

\section{Introduction}

Evolution caused by natural selection can be clearly broken into two steps for purposes of estimation: the effect of selection on phenotypic distributions within a generation and the genetic response of populations across generations (Lande \& Arnold, 1983; Endler, 1986). In a long-term study of the evolution and maintenance of sexual size dimorphism, Preziosi and Fairbairn have documented phenotypic selection acting on male and female body size and components of body size in the water strider Aquarius remigis, both through cross-sectional studies of many populations (Fairbairn, 1988b; Fairbairn \& Preziosi, 1994, 1996) and longitudinal studies of a single population (Preziosi \& Fairbairn, 1996, 1997). In this paper we are concerned with the second step in the estimation of natural selection, the quantitative genetic basis of the morphological traits previously demonstrated to be under phenotypic selection.

If we consider single traits in isolation, the response to selection $(r)$ is simply the product of phenotypic selection $(s)$ on the trait and the herita-

*Correspondence. E-mail: preziosi@ceeb.uky.edu bility of the trait $\left(h^{2}\right) ; r=h^{2} s$ (Falconer, 1989). The effect of the genetic term, heritability, in this model is to reduce the effect of phenotypic selection. When multiple traits are considered, the quantitative genetic model of response for each trait is

$\Delta z i=\beta_{i} h_{i}^{2}+\sum_{j=1}^{n} \beta_{j} h_{j} h_{i} r_{i j}$

for $n$ traits and $i \neq j$ (Lande, 1979; Grant \& Grant, 1995). The first term represents the response to direct selection on trait $i$ whereas the second term represents the indirect response of trait $i$ to direct selection acting on genetically correlated traits included in the model. The $\beta$ values are the partial selection coefficients (or selection gradients sensu Lande \& Arnold [1983]) and estimate direct selection acting on each trait independently of the indirect effects of selection acting on other traits in the model, $h^{2}$ and $h$ are heritability and its square root, respectively, and $r$ is the genetic correlation. The multiple-trait model can also be written using matrix methods as $\Delta \mathbf{z}=\mathbf{G} \boldsymbol{\beta}$, where the change in the vector of trait means $(\boldsymbol{\Delta} \mathbf{z})$ is the product of the genetic covariance matrix $(\mathbf{G}$, which contains the 
combined information of the heritabilities and correlations) and a vector of the partial selection coefficients ( $\beta$ ) (Lande, 1979; Lande \& Arnold, 1983). The effect of the heritabilities and genetic correlations (or the genetic covariance matrix) includes both the reducing effect found in the single-trait model (owing to heritabilities) plus the effects of genetic correlations (or covariance) between traits. The vector of partial selection coefficients $(\boldsymbol{\beta})$ describes the evolutionary trajectory that will produce the maximum rate of increase in mean population fitness (Lande, 1979; Cheverud, 1984). However, evolution proceeds along a trajectory determined jointly by the partial selection coefficients and the genetic parameters (Cheverud, 1984). This deviation from the trajectory of greatest fitness increase is caused by the effects of genetic correlations between traits and it is in this sense that genetic correlations are said to constrain the response to selection (Cheverud, 1984). If these effects are strong enough, individual traits may appear to evolve maladaptively, because of the correlated response to selection acting on other traits (Lande, 1979). Thus, multivariate models are necessary to predict both the rate and direction of response when selection acts on several traits simultaneously. Although the effects of genetic correlations are usually only considered as constraining response to selection, they will also act to constrain evolution that occurs through simple genetic drift (Cheverud, 1984).

Knowledge of the heritabilities and genetic correlations is thus necessary from two related perspectives. First, it is required for the estimation of response to selection, a major goal of evolutionary research (Grant \& Grant, 1995), and secondly, it provides information as to how the response to phenotypic selection may be genetically enhanced or constrained. With respect to the latter point, it is important to examine the genetic matrices for information concerning integration (or relatedness) of traits (sensu Cheverud et al., 1983). Specifically, there may be sets of traits which are highly genetically integrated, and thus likely to evolve together (Lande, 1980; Cheverud et al., 1983).

The water strider Aquarius remigis shows varying degrees and directions of sexual dimorphism for components of body size. Female $A$. remigis are about $10 \%$ longer than males in terms of total length, whereas femoral lengths are not dimorphic, thorax length is only slightly larger in females, abdomen length is $80 \%$ larger in females and genital length and fore-femur width are larger in males (Fairbairn, 1992; Preziosi \& Fairbairn, 1996). This varying pattern of dimorphism suggests that selection is acting on components of body size rather than on body size itself, and targeted selection has been demonstrated several times in this species (Fairbairn \& Preziosi, 1994, 1996; Preziosi \& Fairbairn, 1996, 1997). In addition, Fairbairn (1992) demonstrated that, to some degree, sexual differences in components of body size were independent of the overall difference in total length. The evidence that differences in the dimorphism of components are not a simple scaling effect and that contemporary selection is targeted at some of these components, suggests that the variability in the dimorphism of components of body size may be reflected in the genetic integration of these traits. In this paper we estimate heritabilities and phenotypic and genetic correlations for both male and female A. remigis using a standard half-sib design. We also compare correlation matrices within and between the sexes and examine the genetic matrices for evidence of integration.

\section{Methods}

Aquarius remigis were collected from a stream near Mont-St-Bruno, Quebec $\left(45^{\circ} 39^{\prime} \mathrm{N}, 72^{\circ} 33^{\prime} \mathrm{W}\right)$ in September 1991, and overwintered en masse in plastic containers using the protocol of Fairbairn (1988a). When removed from diapause, females were placed in separate plastic containers $(37 \mathrm{~cm}$ by $25 \mathrm{~cm}$ ) filled to a depth of $6 \mathrm{~cm}$ with tap water and equipped with an airstone. All water striders were kept at $20 \pm 2^{\circ} \mathrm{C}$ and a 12:12 photoperiod. Each container was supplied with half of a styrofoam cup and foam blocks as resting and oviposition sites. Females received half of a frozen mealworm (Tenebrio sp.) and six frozen Drosophila melanogaster per day.

The breeding design was a standard half-sib design with 40 sires and three dams per sire. Each of the 120 females was mated to one of 40 males by placing the male in a female's container for one day in turn (i.e. each female had a male present on every third day). Eggs were counted daily and transferred to a separate container in the incubator. To maintain a balanced design, sire families in which any of the females were producing few or no eggs were discarded and a replacement sire family was set up. Offspring were maintained under the same conditions as their parents. Containers were checked daily and any individuals that had eclosed as adults were frozen at $-20^{\circ} \mathrm{C}$. To reduce bias arising from a small number of offspring per half-sib family (Roff \& Preziosi, 1994), we used only those half-sib 
families that produced at least four male and four female offspring.

Offspring were measured using a computer digitizing system attached to a dissecting microscope. For each individual we made the following measurements: total length, thorax length, abdomen length, genital length, fore-femur width, fore-femur length, mid-femur length and hind-femur length. Figures and detailed descriptions indicating the landmarks of these measurements are presented in Preziosi \& Fairbairn (1996) for males and Preziosi \& Fairbairn (1997) for females.

Heritabilities and genetic correlations were calculated according to standard half-sib methods (Falconer, 1989; Becker, 1992) and are based on the sire estimates of genetic (co)variance. Because of the possible bias caused by a small number of offspring per family (Roff \& Preziosi, 1994) we also calculated jackknife estimates of heritabilities and genetic correlations. Because the estimates from the two methods were very similar, we present only the jackknife estimates here. The methods for using the jackknife in the estimation of heritabilities and genetic correlations are given in Simons \& Roff (1994) and Roff \& Preziosi (1994) with the modification here that pseudovalues were obtained by dropping entire sire families (Roff, 1997). Phenotypic correlations were calculated using the formulae from Becker (1992). Because it has been argued that genetic and phenotypic correlations should be similar when sample sizes are large (Cheverud, 1984, 1988; Koots \& Gibson, 1994; Roff, 1995) we compare genetic and phenotypic correlations within males and females using both simple correlations and Mantel's test (Kohn \& Atchley, 1988; Manly, 1991). Although multiple estimates are made for both phenotypic and genetic values, we have not corrected for this because standard correction procedures are not appropriate for correlated estimates (Manly, 1991) and because we are interested in the general patterns rather than specific estimates.

We also used several methods to determine and compare the structure of the genetic correlation matrices. Principal components analysis of the genetic correlation matrix was used to determine the existence of genetically independent subsets of traits within males and females (Lande, 1979). The existence of such subsets would imply that these groups of traits could respond to selection partially independently of changes within other groups of traits. The 'shape' (sensu Goodnight \& Schwartz, 1997; see this reference for a discussion of matrix comparisons) of male and female genetic correlation matrices was compared using a simple correlation and Mantel's test (Kohn \& Atchley, 1988; Cheverud et al., 1989). To compare the degree of integration within males and females we calculated both the geometric mean of the genetic correlations and Cheverud et al.'s (1983) index of integration (I) which ranges from 0 (no correlations among traits) to 1 (perfect correlations among traits) (Kohn \& Atchley, 1988).

\section{Results}

As expected for morphological traits, the heritabilities (Mousseaau \& Roff, 1987) and genetic correlations (Lande, 1980; Roff \& Fairbairn, 1993; Roff, 1996) were generally high (Tables 1 and 2). Male total length was highly heritable $\left(h^{2} \pm \mathrm{SE}: 1.07\right.$ \pm 0.23 ) and the heritabilities of all other traits except genital length were significantly greater than zero (Table 1). Heritability estimates ranged from

Table 1 Estimates of heritabilities, genetic and phenotypic correlations in male Aquarius remigis. Heritabilities are on the diagonal, genetic correlations are below the diagonal and phenotypic correlations are above the diagonal. Bold values are more than 2 SE from zero

\begin{tabular}{lccccccr}
\hline & $\begin{array}{c}\text { Abdomen } \\
\text { length }\end{array}$ & $\begin{array}{c}\text { Thorax } \\
\text { length }\end{array}$ & $\begin{array}{c}\text { Genital } \\
\text { length }\end{array}$ & $\begin{array}{c}\text { Hind-femur } \\
\text { length }\end{array}$ & $\begin{array}{c}\text { Mid-femur } \\
\text { length }\end{array}$ & $\begin{array}{c}\text { Fore-femur } \\
\text { length }\end{array}$ & $\begin{array}{c}\text { Fore-femur } \\
\text { width }\end{array}$ \\
\hline Abdomen length & $\mathbf{1 . 2 4}$ & $\mathbf{0 . 3 9}$ & -0.02 & $\mathbf{0 . 2 2}$ & $\mathbf{0 . 2 6}$ & $\mathbf{0 . 3 2}$ & $\mathbf{0 . 3 0}$ \\
Thorax length & $\mathbf{0 . 9 9}$ & $\mathbf{1 . 1 6}$ & -0.02 & $\mathbf{0 . 3 7}$ & $\mathbf{0 . 4 7}$ & $\mathbf{0 . 4 6}$ & $\mathbf{0 . 4 6}$ \\
Genital length & -0.07 & 0.28 & 0.11 & -0.06 & -0.05 & 0.00 & -0.02 \\
Hind-femur length & $\mathbf{0 . 8 0}$ & $\mathbf{0 . 9 2}$ & 0.31 & $\mathbf{0 . 3 8}$ & $\mathbf{0 . 5 9}$ & $\mathbf{0 . 5 1}$ & $\mathbf{0 . 3 0}$ \\
Mid-femur length & $\mathbf{0 . 9 1}$ & $\mathbf{1 . 0 2}$ & 0.31 & $\mathbf{0 . 9 9}$ & $\mathbf{0 . 4 6}$ & $\mathbf{0 . 5 4}$ & $\mathbf{0 . 3 6}$ \\
Fore-femur length & $\mathbf{0 . 7 8}$ & $\mathbf{0 . 9 1}$ & 0.11 & $\mathbf{1 . 1 4}$ & $\mathbf{1 . 0 4}$ & $\mathbf{0 . 7 2}$ & $\mathbf{0 . 4 1}$ \\
Fore-femur width & $\mathbf{0 . 9 6}$ & $\mathbf{0 . 9 6}$ & 0.49 & $\mathbf{0 . 7 0}$ & $\mathbf{0 . 7 5}$ & $\mathbf{0 . 7 7}$ & $\mathbf{0 . 6 9}$ \\
\hline
\end{tabular}


Table 2 Estimates of heritabilities, genetic and phenotypic correlations in female Aquarius remigis. Heritabilities are on the diagonal, genetic correlations are below the diagonal and phenotypic correlations are above the diagonal. Bold values are more than $2 \mathrm{SE}$ from zero

\begin{tabular}{lccccc}
\hline & $\begin{array}{c}\text { Abdomen } \\
\text { length }\end{array}$ & $\begin{array}{c}\text { Thorax } \\
\text { length }\end{array}$ & $\begin{array}{c}\text { Hind-femur } \\
\text { length }\end{array}$ & $\begin{array}{c}\text { Mid-femur } \\
\text { length }\end{array}$ & $\begin{array}{c}\text { Fore-femur } \\
\text { width }\end{array}$ \\
\hline Abdomen length & $\mathbf{0 . 6 3}$ & $\mathbf{0 . 5 4}$ & $\mathbf{0 . 3 7}$ & $\mathbf{0 . 3 9}$ & $\mathbf{0 . 3 3}$ \\
Thorax length & $\mathbf{0 . 7 0}$ & $\mathbf{0 . 8 4}$ & $\mathbf{0 . 4 6}$ & $\mathbf{0 . 4 8}$ & $\mathbf{0 . 3 8}$ \\
Hind-femur length & $\mathbf{0 . 4 6}$ & $\mathbf{0 . 9 8}$ & $\mathbf{0 . 7 0}$ & $\mathbf{0 . 6 4}$ & $\mathbf{0 . 2 7}$ \\
Mid-femur length & 0.44 & $\mathbf{0 . 8 8}$ & $\mathbf{1 . 0 2}$ & $\mathbf{0 . 8 8}$ & $\mathbf{0 . 2 7}$ \\
Fore-femur width & $\mathbf{0 . 7 1}$ & $\mathbf{0 . 7 1}$ & $\mathbf{0 . 5 1}$ & 0.40 & $\mathbf{0 . 2 4}$ \\
\hline
\end{tabular}

0.11 to 1.24 with the average heritability (0.68) falling within the range expected for morphological traits (Mousseau \& Roff, 1987). Genetic correlations between male traits ranged from -0.07 to 1.14 and phenotypic correlations ranged from -0.06 to 0.59 . Excluding correlations with genital length, which were all nonsignificant, genetic correlations were all positive and large. A plot of genetic vs. phenotypic correlations (Fig. 1, top panel) indicates that, although the pattern of correlations was similar, the phenotypic correlations consistently underestimated the magnitude of the genetic correlations. Although the average phenotypic correlation (0.28) was smaller than the average genetic correlation (0.72), the patterns of the phenotypic and genetic correlation matrices were highly similar $(r=0.902$, $P<0.001$; Mantel's test, $P<0.01)$.

Total length in females was also highly heritable $(0.68 \pm 0.19)$. Estimates of phenotypic and genetic parameters for other traits of females are given in Table 2. Variance components for fore-femur length and genital length of females were negative (probably resulting from estimation error) and thus heritabilities and genetic correlations could not be calculated for these traits. Heritabilities of the remaining traits were all significantly greater than zero and ranged from 0.24 to 0.88 . The average heritability (0.66) was high and very similar to the average heritability for males (0.68). The majority of genetic correlations $(80 \%)$ and all of the phenotypic correlations were significantly greater than zero. The genetic correlations ranged from 0.40 to 1.02 and phenotypic correlations ranged from 0.27 to 0.64 . As in males, the average phenotypic correlation $(0.41)$ was smaller than the average genetic correlation (0.68) but the structure of the genetic and phenotypic correlation matrices was similar $(r=0.76$, $P<0.01$; Mantel's test, $P<0.05)$. A plot of the genetic vs. phenotypic correlations for females (Fig. 1, bottom panel) indicates that, as in males, phenotypic correlations consistently underestimated genetic correlations.

Genetic correlations between males and females were generally positive (Table 3 ). Total length was highly correlated between the sexes $\left(r_{\mathrm{A}} \pm \mathrm{SE}\right.$ : $0.86 \pm 0.17)$. Female abdomen length, thorax length and fore-femur width also tended to be highly and significantly positively correlated with all male traits. Femoral length traits of females were not significantly correlated with traits in males except for male fore-femur width which was significantly negatively correlated. For correlations between the same trait in males and females (values on the diagonal in Table 3), abdomen length, thorax length and forefemur width showed high positive correlations whereas femur lengths were not significantly correlated between the sexes.

Loadings from the PCA of the within-sex genetic correlation matrices (Table 4) indicated strong genetic integration of components within both males and females. For both males and females the first PC accounted for $75-80 \%$ of the additive genetic covariance and all traits weighed positively on these first components. The second PC in males clearly showed a separation of fore-femur width and genital length from other male traits. Although the second PC in males accounted for only $16 \%$ of the additive genetic covariance, the separation along this PC defined two genetic groups of traits which can respond to selection in a partially independent manner. The second PC for females accounted for $19 \%$ of the additive genetic covariance and suggests a genetic separation of abdomen length and forefemur width from femur lengths. As in males, this suggests that these two groups of traits are partially genetically independent in their response to selec- 
tion. Both the integration index and the geometric mean of the genetic correlations was higher in females $(I=0.625$, geometric mean $r=0.418)$ than in males $(I=0.581$, geometric mean $r=0.359)$. Unfortunately, no method is available to test for differences in these values (Cheverud et al., 1983) but, based on the standard errors of the individual correlations, it seems unlikely that such a small difference would be significant. Both a standard correlation among the elements of the genetic correlation matrices $(r=0.715, P<0.05)$ and Mantel's test
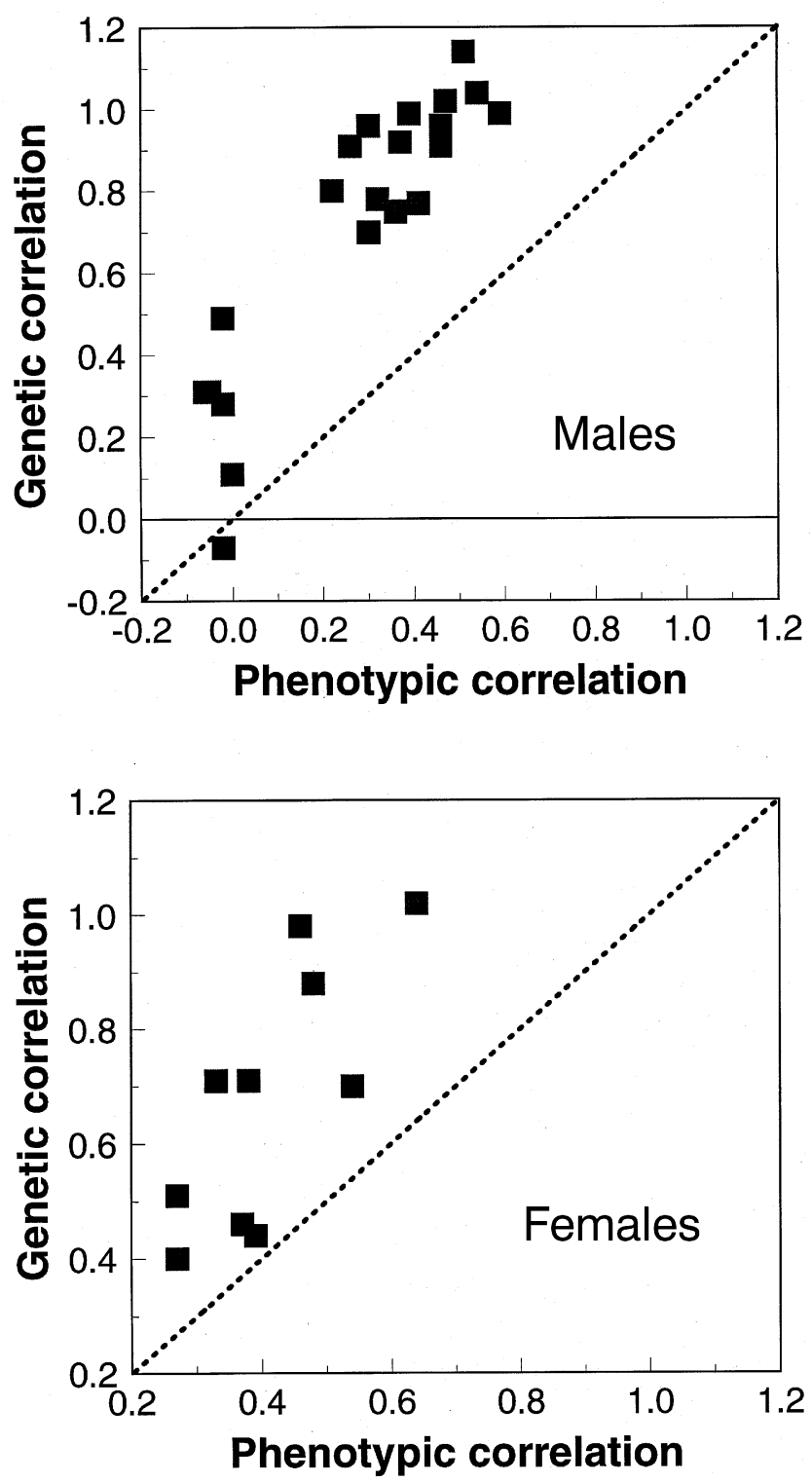

Fig. 1 Within-sex genetic vs. phenotypic correlations for male and female Aquarius remigis.
$(P<0.05)$ indicated that the pattern of the genetic correlation matrix was similar in males and females.

\section{Discussion}

Estimates of selection in natural populations of $A$. remigis have consistently demonstrated sexual selection for larger male body size (Fairbairn, 1988b; Fairbairn \& Preziosi, 1994) and fecundity selection for larger female body size (Fairbairn, 1988b; Blanckenhorn, 1994; Preziosi \& Fairbairn, 1997). Lifetime estimates of selection on adult body size found that survival selection favoured smaller total length in both males and females and that total length in both sexes was under lifetime stabilizing selection (Preziosi \& Fairbairn, 1996, 1997). The high heritability of total length for both males and females indicates that total length will show a response to the phenotypic selection we have previously demonstrated (Preziosi \& Fairbairn, 1996, 1997) and suggests that contemporary selection is acting to maintain sexual size dimorphism in this species. The high positive genetic correlation between the sexes is typical for traits expressed in both sexes (Lande, 1980; Roff \& Fairbairn, 1993) and, although slowing the rate at which the individual sexes reach their optima, should not prevent the eventual attainment of equilibrium values of male and female body size (Lande, 1980).

Recent papers have demonstrated that, at least for morphological traits, laboratory estimates of genetic parameters can reasonably be extrapolated to field situations. The common concern is that the reduced environmental variance in the laboratory will lead to overestimation of the genetic contribution in the field (i.e. inflated heritabilities and genetic correlations) (Blum, 1988; Simons \& Roff, 1996). Weigensberg \& Roff (1997) reviewed studies where both laboratory and field heritabilities had been estimated and found no significant difference between laboratory and field estimates for morphological, life history or behavioural traits. Similarly, Simons \& Roff (1996) compared laboratory and field estimates of genetic correlations among several morphological and one life history trait of a field cricket. They found a strong agreement between laboratory and field estimates $(r=0.97)$ for morphological traits but weak agreement for the life history variable. Fairbairn (1990) provides estimates of phenotypic variance for total length of males and females from 12 field-caught populations (mean of 0.215 for males and 0.167 for females) and five laboratory-reared samples (mean of 0.342 for males and 0.374 for females) of $A$. remigis. Although the 
Table 3 Genetic correlations between male and female traits in Aquarius remigis. Bold values are more than 2 SE from zero

\begin{tabular}{llccccc}
\hline & \multicolumn{5}{c}{ Female } \\
\cline { 3 - 6 } & $\begin{array}{c}\text { Abdomen } \\
\text { length }\end{array}$ & $\begin{array}{c}\text { Thorax } \\
\text { length }\end{array}$ & $\begin{array}{c}\text { Hind-femur } \\
\text { length }\end{array}$ & $\begin{array}{c}\text { Mid-femur } \\
\text { length }\end{array}$ & $\begin{array}{c}\text { Fore-femur } \\
\text { width }\end{array}$ \\
\hline Male & Abdomen length & $\mathbf{1 . 0 1}$ & $\mathbf{1 . 1 1}$ & 0.20 & 0.11 & $\mathbf{0 . 8 7}$ \\
& Thorax length & $\mathbf{0 . 6 5}$ & $\mathbf{0 . 7 7}$ & 0.16 & -0.03 & $\mathbf{0 . 8 0}$ \\
& Hind-femur length & $\mathbf{1 . 3 6}$ & $\mathbf{0 . 9 4}$ & 0.36 & 0.41 & 0.77 \\
& Mid-femur length & $\mathbf{1 . 4 1}$ & $\mathbf{0 . 8 9}$ & 0.14 & 0.17 & $\mathbf{0 . 7 9}$ \\
& Fore-femur width & $\mathbf{0 . 8 0}$ & 0.30 & $-\mathbf{0 . 3 8}$ & $-\mathbf{0 . 4 8}$ & $\mathbf{0 . 8 9}$ \\
\hline
\end{tabular}

field and laboratory variances were not significantly different for either males or females, the laboratory estimates of phenotypic variance were consistently higher. Thus it seems unlikely that our laboratory estimates of genetic parameters are inflated because of a reduction in environmental variance. For the females used in this study the phenotypic variance of the parental females was 1.4 times that of their laboratory-reared offspring (see Roff, 1997, fig. 2.14). If we assume no genotype-by-environment interaction, the laboratory estimate of heritability of female total length $(0.68)$ would overestimate the field value $(0.68 / 1.4=0.49)$ but, based on the SE of the laboratory estimate (0.19), this difference would be far from significant. Thus, laboratory estimates for this species can reasonably be extrapolated to the field.

This study also addresses the hypothesis that phenotypic correlations are reasonable substitutes

Table 4 Component loadings for the first and second principal components from male and female genetic correlation matrices for Aquarius remigis. Loadings are only provided for components explaining more variance than an original variable

\begin{tabular}{lccccc}
\hline & \multicolumn{2}{c}{ Males } & & \multicolumn{2}{c}{ Females } \\
\cline { 2 - 3 } \cline { 6 - 6 } & 1 & 2 & & 1 & 2 \\
\hline Abdomen length & 0.924 & 0.299 & & 0.741 & -0.550 \\
Thorax length & 1.005 & 0.012 & & 0.994 & 0.057 \\
Genital length & 0.297 & -0.966 & & - & - \\
Hind-femur length & 0.967 & 0.019 & & 0.940 & 0.383 \\
Mid-femur length & 0.994 & 0.025 & & 0.888 & 0.450 \\
Fore-femur length & 0.972 & 0.158 & & - & - \\
Fore-femur width & 0.900 & -0.220 & & 0.747 & -0.547 \\
Per cent variance & 80.40 & 15.68 & & 75.35 & 19.09 \\
\hline
\end{tabular}

(C) The Genetical Society of Great Britain, Heredity, 81, 92-99. for genetic correlations (Cheverud, 1988; Roff, 1995, 1996). Although there was a strong similarity in the pattern (shape) of the genetic and phenotypic correlation matrices, the phenotypic correlations are consistently lower than the genetic correlations. Thus, for $A$. remigis, substitution of the phenotypic for the genetic correlations would underestimate correlated response, i.e. overestimate the degree to which traits can independently respond to selection.

Using the multivariate regression methods of Lande \& Arnold (1983) applied to the same set of morphological traits examined in this study, selection on total length has been shown to be acting on specific components of body size (hereafter targets of selection) rather than total length itself for both male and female $A$. remigis. Studies of natural populations have revealed opposing selection acting on components of body size (Fairbairn \& Preziosi, 1996; Preziosi \& Fairbairn, 1996) and the generally high and positive genetic correlations among components of body size both within and between the sexes will act to constrain response to this opposing selection. For females, both fecundity selection and survival selection on total length appear to target abdomen length (Preziosi \& Fairbairn, 1997). The high heritability of female abdomen length and the high genetic correlations with other traits indicate that all components of female body size will respond strongly to phenotypic selection on abdomen length. Within males, genital length is an exception to the general pattern in the genetic matrix, with both heritability and genetic correlations being nonsignificant. Thus, although sexual selection for larger males targets genital length (Preziosi \& Fairbairn, 1996), genital length itself will not respond, or respond only weakly, to this selection. This reduction in additive genetic variance in genital length is possibly the result of strong selection on this trait as expected from the standard quantitative genetic 
model. However, abdomen length in females, also a consistent target of selection, shows no such reduction in genetic variance. Interestingly, total length of males is under selection and highly heritable and, as longer males do have longer genitals both phenotypically $(r=0.59)$ and genetically $(r=0.45)$, genital length will show a correlated response to this selection. Although traits under selection are expected to lose additive genetic variance (Fisher, 1958), morphological (and other) traits generally have nonzero heritabilities (Mousseau \& Roff, 1987), an observation that has inspired several hypotheses for the maintenance of genetic variance. The pattern of selection and genetic parameters in male $A$. remigis suggests a simple explanation for the nonzero genetic variation of male total length in this species. Genetic variance of total length may be eroded at a much lower rate than the selection on total length implies, simply because selection is actually targeted at another, positively correlated, trait (genital length); a trait which does show a reduction in genetic variance.

The genetic correlations between the sexes are difficult to interpret in isolation because the response to selection depends on the combined effects of the within- and between-sex genetic matrices. However, the simple expectation that genetic correlations between dimorphic traits will be reduced (Lande, 1980) does not seem to be borne out in this study. Both abdomen length and forefemur width are dimorphic but have the highest correlations between the sexes. Although we generally expect positive correlations between the sexes (at least for same-trait correlations [Lande, 1980; Roff \& Fairbairn, 1993]), the pattern of correlations between the sexes in $A$. remigis indicates that leg length in females has become genetically disassociated from most traits in males and negatively associated with male fore-femur width.

The first principal component of the genetic correlation matrices, the index of integration and the average genetic correlation all indicate that both males and females have a high overall genetic integration for the morphological traits that we examined. Comparison of the within-sex genetic correlations suggests that the pattern of covariance, and thus the pattern of integration, is similar in the sexes. However, the PCA of the genetic matrix also clearly identified subgroups of traits which can show a partially independent response to selection. PC2 in males appears to separate traits based on their pattern of sexual dimorphism (i.e. male- or femalebiased). The strongest separation in males is between genital length and abdomen length. These two traits show the strongest sexual dimorphism of the traits examined, with abdomen length much larger in females and genital length much larger in males (Fairbairn, 1992; Preziosi \& Fairbairn, 1996). Females also show a separation of dimorphic and monomorphic traits on PC2, although in this case the separation does not appear to be related to the direction of dimorphism as abdomen length is female-biased and fore-femur width is male-biased. The pattern of isolation in males and females suggests that the selection favouring the evolution of sexual dimorphism has altered the genetic matrix to isolate, at least partially, groups of traits under selection for dimorphism. This isolation may also be reflected in the genetic correlations between the sexes, although it is difficult to interpret the impact of single elements in the between-sex matrix. The fact that fore-femur width is partially isolated within both sexes (PCA), partially isolated in the betweensex matrix, and does not appear to be under contemporary selection for larger size (Fairbairn \& Preziosi, 1996; Preziosi \& Fairbairn, 1996), suggests that the dimorphism for fore-femur width may be a historical genetic effect.

Documenting the effects of selection in natural populations requires the estimation of both phenotypic selection and the genetic parameters which produce a response to that selection. The high heritabilities for total length and several components of body size in $A$. remigis suggest that we should see a strong response to the selection we have documented in natural populations of this species, selection which acts to maintain sexual size dimorphism for total length. However, the general pattern of high positive genetic correlations, both within and between the sexes, will act as a constraint to the opposing selection acting on components of body size. The pattern of genetic correlations within the sexes and the PCA suggest that the genetic correlation matrix has been altered in response to selection for sexual dimorphism of body size components to minimize the correlated response among dimorphic and monomorphic traits within each sex. Previous models of the evolution of SSD have usually examined only single traits and have suggested that selection for dimorphism will reduce the genetic correlation between the sexes [although this reduction is not required for traits to reach equilibrium (Lande, 1980)]. The pattern we observe here for multiple traits, some with opposing directions of sexual dimorphism, suggests that selection may have acted to isolate dimorphic traits genetically within each sex rather than reduce the between-sex genetic correlations both between dimorphic and mono- 
morphic traits and among dimorphic traits themselves.

\section{References}

BECKER, w. A. 1992. Manual of Quantitative Genetics, 5th edn. Academic Enterprises, Pullman, WA.

BLANCKENHORN, W. U. 1994. Fitness consequences on alternative life histories in water striders, Aquarius remigis (Heteroptera: Gerridae). Oecologia, 97, 354-365.

Blum, A. 1988. Plant Breeding for Stress Environments. CRC Press, Boca Raton, FL.

CHEVERUD, J. M. 1984. Quantitative genetics and developmental constraints on evolution by selection. J. Theor. Biol., 110, 155-171.

CHEVERUd, J. M. 1988. A comparison of genetic and phenotypic correlations. Evolution, 42, 958-968.

CHEVERUD, J. M., RUTLEDGe, J. J. AND ATCHLEY, W. R. 1983. Quantitative genetics of development: genetic correlations among age-specific trait values and the evolution of ontogeny. Evolution, 37, 895-905.

CHEVERUd, J. M., WAGNER, G. P. AND DOW, M. M. 1989. Methods for the comparative analysis of variation patterns. Syst. Zool., 38, 201-213.

ENDLeR, J. A. 1986. Natural Selection in the Wild. Princeton University Press, Princeton, NJ.

FAIRBAIRN, D. J. 1988a. Adaptive significance of wing dimorphism in the absence of dispersal: a comparative study of wing morphs in the waterstrider Gerris remigis. Ecol. Entomol., 13, 273-281.

FAIRBAIRN, D. J. 1988b. Sexual selection for homogamy in the Gerridae: an extension of Ridley's comparative approach. Evolution, 42, 1212-1222.

FAIRBAIRN, D. J. 1990. Factors influencing sexual size dimorphism in temperate waterstriders. Am. Nat., 136, 61-86.

FAIRBAIRN, D. J. 1992. The origins of allometry: size and shape polymorphism in the common waterstrider, Gerris remigis Say (Heteroptera: Gerridae). Biol. J. Linn. Soc., 45, 167-186.

FAIRBAIRN, D. J. AND PREZIOSI, R. F. 1994. Sexual selection and the evolution of allometry for sexual size dimorphism in the water strider, Aquarius remigis. Am. Nat., 144, 101-118.

FAIRBAIRN, D. J. AND PREZIOSI, R. F. 1996. Sexual selection and the evolution of sexual size dimorphism in the water strider, Aquarius remigis. Evolution, 50, $1549-1559$.

FALCONER, D. S. 1989. Introduction to Quantitative Genetics, 3rd edn. John Wiley and Sons, New York.

FISHER, R. A. 1958. The Genetical Theory of Natural Selection, 2nd edn. Dover, New York.

GOODNIGHT, C. J. AND SCHWARTZ, J. M. 1997. A bootstrap comparison of genetic covariance matrices. Biometrics,
53, 346-359.

GRANT, P. R. AND GRANT, B. R. 1995. Predicting microevolutionary responses to directional selection on heritable variation. Evolution, 49, 241-251.

KOHN, L. A. P. AND ATCHLEY, W. R. 1988. How similar are genetic correlation structures? Data from mice and rats. Evolution, 42, 467-481.

KOOTS, K. R. AND GIBSON, J. P. 1994. How precise are genetic correlation estimates? In: Smith, C. et al. (eds) Proc. 5th World Cong. Gen. Appl. Livestock Prod., 18, 353-356. University of Guelph, Guelph, Ontario.

LANDE, R. 1979. Quantitative genetic analysis of multivariate evolution, applied to brain:body size allometry. Evolution, 33, 402-416.

LANDE, R. 1980. Sexual dimorphism, sexual selection, and adaptation in polygenic characters. Evolution, 34, 292-305.

LANDE, R. AND ARNOLD, s. J. 1983. The measurement of selection on correlated characters. Evolution, 37, $1210-1226$.

MANLY, B. F. J. 1991. Randomization and Monte Carlo Methods in Biology. Chapman and Hall, New York.

MOUSSEAU, T. A. AND ROFF, D. A. 1987. Natural selection and the heritability of fitness components. Heredity, $\mathbf{5 9}$, 181-197.

PREZIOSI, R. F. AND FAIRBAIRN, D. J. 1996. Sexual size dimorphism and selection in the wild in the waterstrider Aquarius remigis: body size, components of body size and male mating success. J. Evol. Biol., 9, 317-336.

PREZIOSI, R. F. AND FAIRBAIRN, D. J. 1997. Sexual size dimorphism and selection in the wild in the waterstrider Aquarius remigis: lifetime fecundity selection on female total length and its components. Evolution, 51, 467-474.

ROFF, D. A. 1995. The estimation of genetic correlations from phenotypic correlations: a test of Cheverud's conjecture. Heredity, 74, 481-490.

ROFF, D. A. 1996. The evolution of genetic correlations: an analysis of patterns. Evolution, 50, 1392-1403.

ROFF, D. A. 1997. Evolutionary Quantitative Genetics. Chapman and Hall, New York.

ROFF, D. A. AND FAIRBAIRN, D. J. 1993. The evolution of alternate morphologies: fitness and wing morphology in male sand crickets. Evolution, 47, 1572-1584.

ROFF, D. A. AND PREZIOSI, R. 1994. The estimation of the genetic correlation: the use of the jackknife. Heredity, 73, 544-548.

SIMONS, A. M. AND ROFF, D. A. 1994. The effect of environmental variability on the heritabilities of traits of a field cricket. Evolution, 48, 1637-1649.

SIMONS, A. M. AND ROFF, D. A. 1996. The effect of a variable environment on the genetic correlation structure in a field cricket. Evolution, 50, 267-275.

WEIGENSBERG, I. AND ROFF, D. A. 1997. Natural heritabilities: can they be reliably estimated in the laboratory? Evolution, 50, 2149-2157. 